\title{
Usefulness of Intraoperative Computed Tomography in Complication Management after Spine Surgery
}

\author{
Johannes Kerschbaumer ${ }^{1} \quad$ Christian Franz Freyschlag ${ }^{1}$ Marcel Seiz-Rosenhagen ${ }^{2}$ Claudius Thomé ${ }^{1}$ \\ ${ }^{1}$ Department of Neurosurgery, Innsbruck Medical University, \\ Innsbruck, Austria \\ 2 Department of Neurosurgery, University Medical Center Mannheim, \\ Mannheim, Germany \\ Address for correspondence Johannes Kerschbaumer, MD, \\ Department of Neurosurgery, Innsbruck Medical University, \\ Anichstrasse 35, A-6020 Innsbruck, Austria \\ (e-mail: johannes.kerschbaumer@tirol-kliniken.at).
}

Indian J Neurosurg 2015;4:193-198.

\begin{abstract}
Intraoperative computed tomographic (CT) scanners are used frequently in spine surgery for exact pedicle screw placement. In complication management, imaging is mainly used outside the operation theater. A 69-year-old female patient with progressive cervical radiculopathy and myelopathy due to spinal stenosis at $C 4 / 5$ and C5/6 underwent two-level anterior decompression and fusion uneventfully. Shortly after surgery the patient developed a rapid loss of motor function in the upper and lower limbs, followed by a rising sensory deficit. A cage dislocation or an epidural hematoma was suspected and immediate surgical decompression was warranted. A $\mathrm{CT}$ scan was performed using an intraoperative $\mathrm{CT}$ right after reintubation. It showed an extensive epidural hematoma ranging from $\mathrm{C} 2$ to $\mathrm{C} 4$. A reoperation and removal of the hematoma was performed via a C5 corpectomy, extended to a C4-C5 corpectomy due to persistent compression demonstrated on a second intraoperative CT. After the revision operation the patient presented with only a minimal paraparesis of the lower limbs, declining over the next 2 weeks. A magnetic resonance imaging scan is usually

Keywords

- ioCT

- spinal

- epidural

- hematoma

- complication requested in case of a new neurological deficit after cervical spine surgery to detect an epidural hematoma or edema of the spinal cord. Normal neurological examination after extubation followed by rapid progressive deterioration is most likely suspicious to be caused by a hematoma compressing the spinal cord. In the presented case, a CT scan in the operation theater provides adequate imaging information at short hand, aiding the surgeon to perform an emergency revision operation.
\end{abstract}

\section{Introduction}

Anterior cervical spine operations involve decompression of the spinal canal and cervical nerve roots by discectomy or corpectomy in combination with fusion via cage or replacement of a vertebral body. Bailey and Badgley, ${ }^{1}$ Smith and Robinson, ${ }^{2}$ and Cloward $^{3}$ introduced the technique first in the 1950s and the early 1960s.

The reported rates of clinical success in the literature are high $^{4}$ and low-complication rates described, such as wound infection (0.4-2\%), cerebral spinal fluid leak (1\%), hematoma (1\%), hoarseness or dysphagia (9\%), nonfusion ${ }^{5}$ or accelerated adjacent disc degeneration (2.9\%). ${ }^{6}$ One of the most serious adverse events associated with cervical spine surgery is a postoperative spinal epidural hematoma (SEH), compressing the cervical spinal cord. Although rates of SEH after lumbar spine surgery are reported in 33 to $100 \%^{7-9}$ on imaging, most of them remain asymptomatic. Reported rates of symptomatic SEH after anterior decompressive cervical spine surgery vary received

September 11, 2015

accepted

September 28, 2015

published online

December 16, 2015
DOI http://dx.doi.org/

10.1055/s-0035-1569003. ISSN 2277-954X.
(C) 2015 Neurological Surgeons' Society of India

License terms

c) $(1) Ð$ 
from 0.1 to $3.2 \%$, slightly elevated in case of corpectomy compared with multilevel discectomy and fusion. ${ }^{10,11}$ If this occurs and is not evacuated immediately, persistent neurological deficits (viz., such as paralysis or even death because of compromised spontaneous breathing) may occur.

Here, we present a case of SEH after two-level discectomy leading to paralysis within an hour postoperatively, and the advantage of an intraoperative CT (ioCT) providing an immediate diagnosis and control of hematoma evacuation.

\section{Case Report}

A 69-year-old female patient presented with cervical radiculopathy and myelopathy. Magnetic resonance imaging (MRI) of the cervical spine revealed a multilevel cervical spondylotic myelopathy (CSM) at C4/5 and C5/6 (-Fig. 1). Anterior decompression and fusion (ACDF) of both levels was performed uneventfully.

After weaning the patient presented without neurological deficit. Within 45 minutes after surgery, deterioration with progressive motor deficit to complete tetraplegia developed in the recovery unit. Because of this dramatic deficit and a rising sensory deficit level during preparation for reintubation, an emergency MRI scan was dismissed. A CT scan in the operation theater with the patient already positioned for anterior cervical surgery was performed instead (11.55 AM) in suspicion of a spinal cord compression due to cage dislocation or hematoma. It revealed a massive ventral hematoma preponderantly behind C3 and C4 with compression of the spinal cord (-Fig. $2 \mathbf{2 a}$ ).

The wound was rapidly reopened, the plate and both cages removed and hematoma was sucked through the intervertebral spaces within less than 30 minutes after the initial scan. Corpectomy of the C5 was performed and a blood clot was removed behind C4 supported by rinsing via an external ventricular drainage catheter until the hematoma seemed to be evacuated adequately. Using the ioCT, another scan was performed 58 minutes after the skin incision and a residual hematoma behind the vertebral body C3 was detected (-Fig. 2b).

The approach was enlarged by additional corpectomy of C4 to ensure an adequate evacuation of the hematoma. The second ioCT (104 minutes after the incision) showed only a small residual hematoma behind $\mathrm{C} 2$ without significant compression of the spinal cord and the dural sac (-Fig. 2c). Osteosynthesis was achieved using a 35-mm meshcage, packed with autograft bone and a ventral fixation with a $45-\mathrm{mm}$ plate.

After prolonged hemostasis and wound closure, a last control scan was performed, showing a sufficient ventral decompression and confirmed correct positioning of the bicortical screws (-Fig. 3). Before reoperation prophylactic intravenous antibiotic and intravenous corticoid was administered.

After extubation the patient presented with physiological movement of the upper and lower limbs and spontaneous breathing. Meticulous testing on the first postoperative day revealed a mild paraparesis (weak 4 out of 5) without a sensory deficit.

Supported by physiotherapeutic and ergotherapeutic treatment, the patient could be mobilized rapidly and she was able to walk alone for short distances after 2 weeks. For further rehabilitation, the patient was transferred to a specialized center, where the neurological deficits further declined. After 1 month of the operation the patient had recovered completely from her major neurological deficits.

\section{Discussion}

ACDF is the primary approach for treating CSM. Common complications such as wound problems, temporary hoarseness, or mild dysphagia range up to $10 \%^{5}$ in patients undergoing ACDF. Symptomatic SEH after cervical discectomy are described with an incidence between 0.1 and $0.2 \%,{ }^{9}$ whereas asymptomatic SEH seem to happen in 30 up to $100 \%$ of patients after lumbar spine surgery. ${ }^{7-9}$ In cervical spine surgery, however, a postoperative SEH becomes symptomatic earlier due to different relation of the neural tissue to the volume of the spinal canal. In case of new neurological deficits immediately after cervical spine surgery, three major differential diagnoses have to be made: either a dislocation of the implant or a postoperative hematoma can compress the spinal cord, leading to a rising motor or sensible deterioration. Also, an ischemic damage to the spinal cord has to be concerned as a potential differential diagnosis.

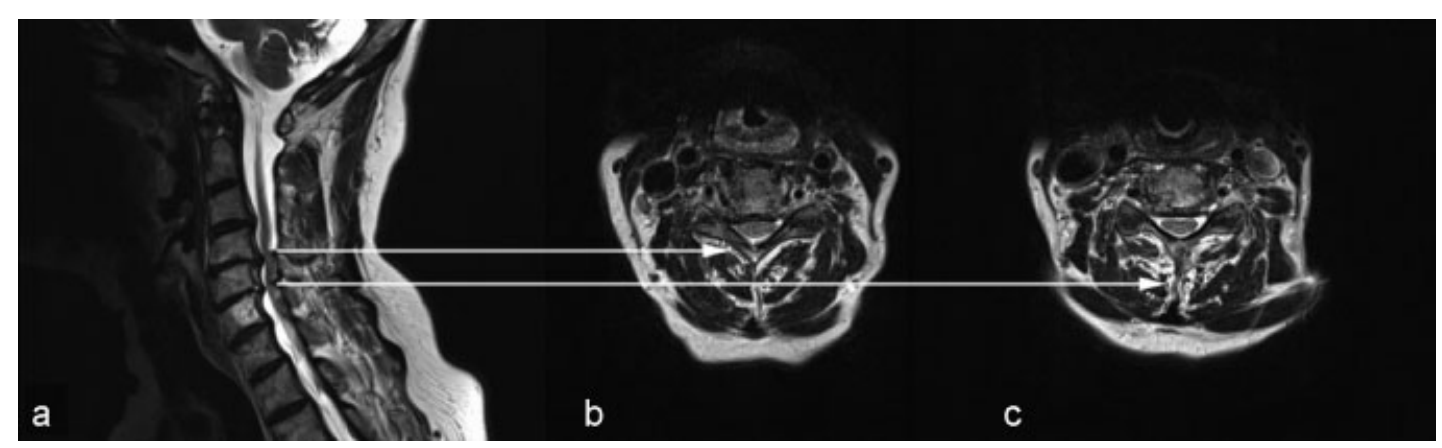

Fig. 1 (a) Sagittal MRI scan showing cervical myelopathy at level C4/5 and C5/6, (b) axial slice at level C4/5, and (c) at level C5/6 with concentric constriction of the spinal canal. MRI, magnetic resonance imaging. 


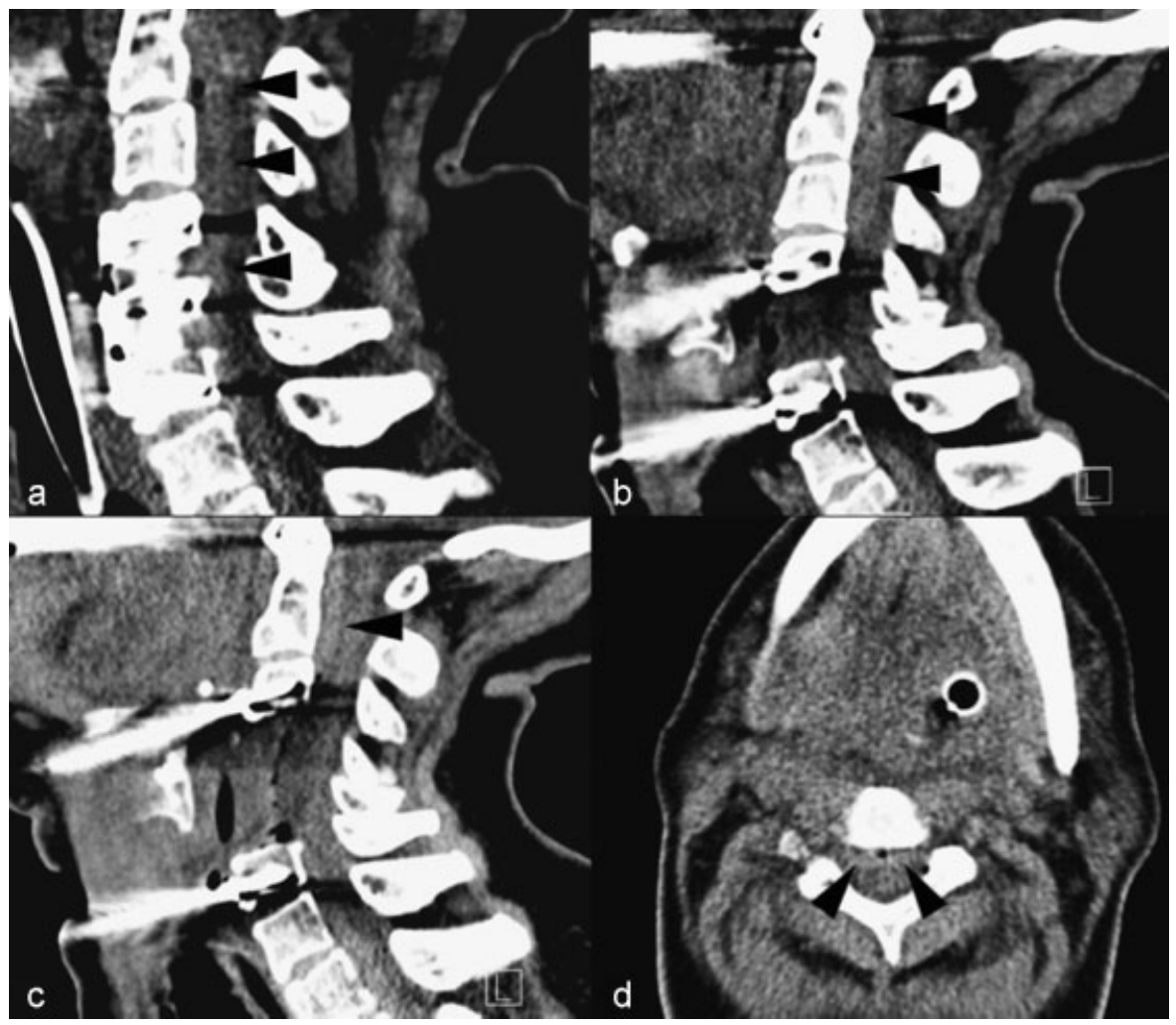

Fig. 2 (a) CT scan 1 hour after two-level ACDF of a patient developing complete tetraplegia. A large hematoma ventral to the dural sac can be seen from C2 to C6, (b) ioCT scan after corpectomy C5 revealing a residual hematoma behind the vertebral body of C2 to C3, (c) second ioCT scan after additional corpectomy of C4, showing minimal residual hematoma behind C2, and (d) not compressing the spinal cord. ACDF, anterior decompression and fusion; $\mathrm{CT}$, computed tomographic, ioCT, intraoperative $\mathrm{CT}$.

Hans et al described a second look operation without follow-up imaging in suspicion of local hematoma after acute deterioration in patients after ACDF. ${ }^{12}$ Hematomas, however, can spread to levels above, and below the level actually operated. In fact, in the presented case we found a hematoma spreading over more than two levels. An adequate decompression would not be possible by removing the implants alone, but a corpectomy had to be performed. The fact that acute compression of the spinal cord by coagulated blood has a poor prognosis, if not removed quickly, affirms the need of adequate and immediate imaging in the case of these complications.

The acute imaging was performed using an ioCT, which delivered precise information, especially in the detection of hematomas. Also, an eventual dislocation of the implants could be excluded quickly. Even if MRI is the most effective diagnostic tool for detecting postoperative $\mathrm{SEH}$ or confirmation of an ischemic damage, it is time-consuming, it can be challenging from a logistical point of view, and it is not always available immediately (-Fig. 4, online-only).

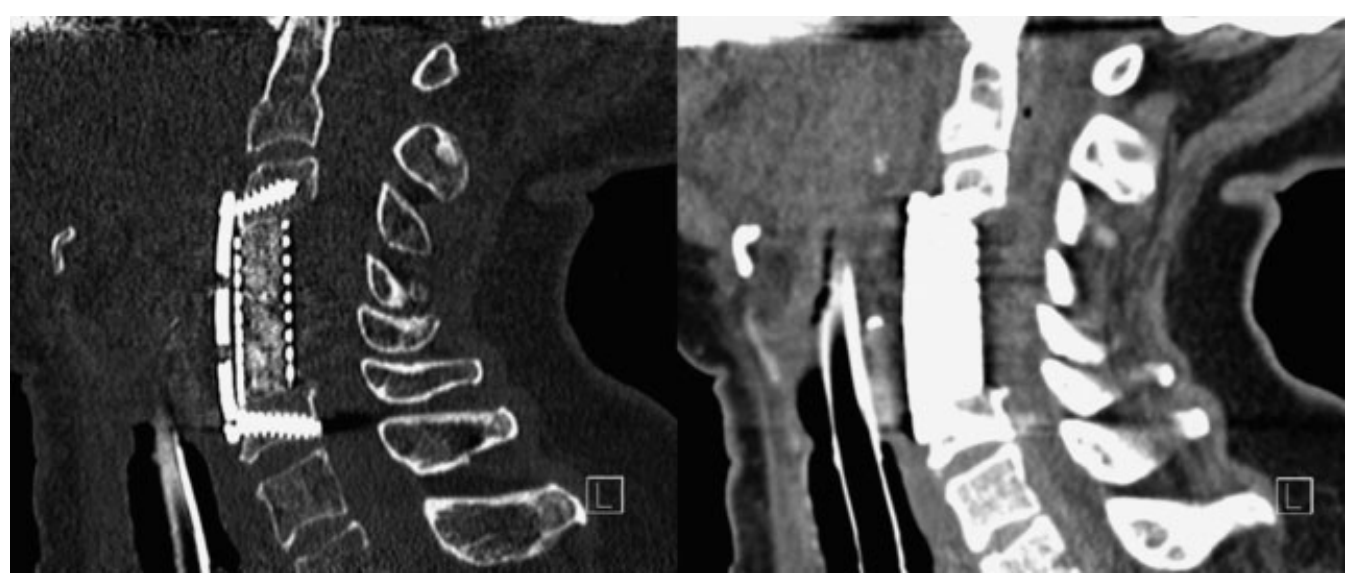

Fig. 3 Postoperative control scan, showing adequate decompression and sufficient anchoring of the bicortical screws in C6 vertebral body. 


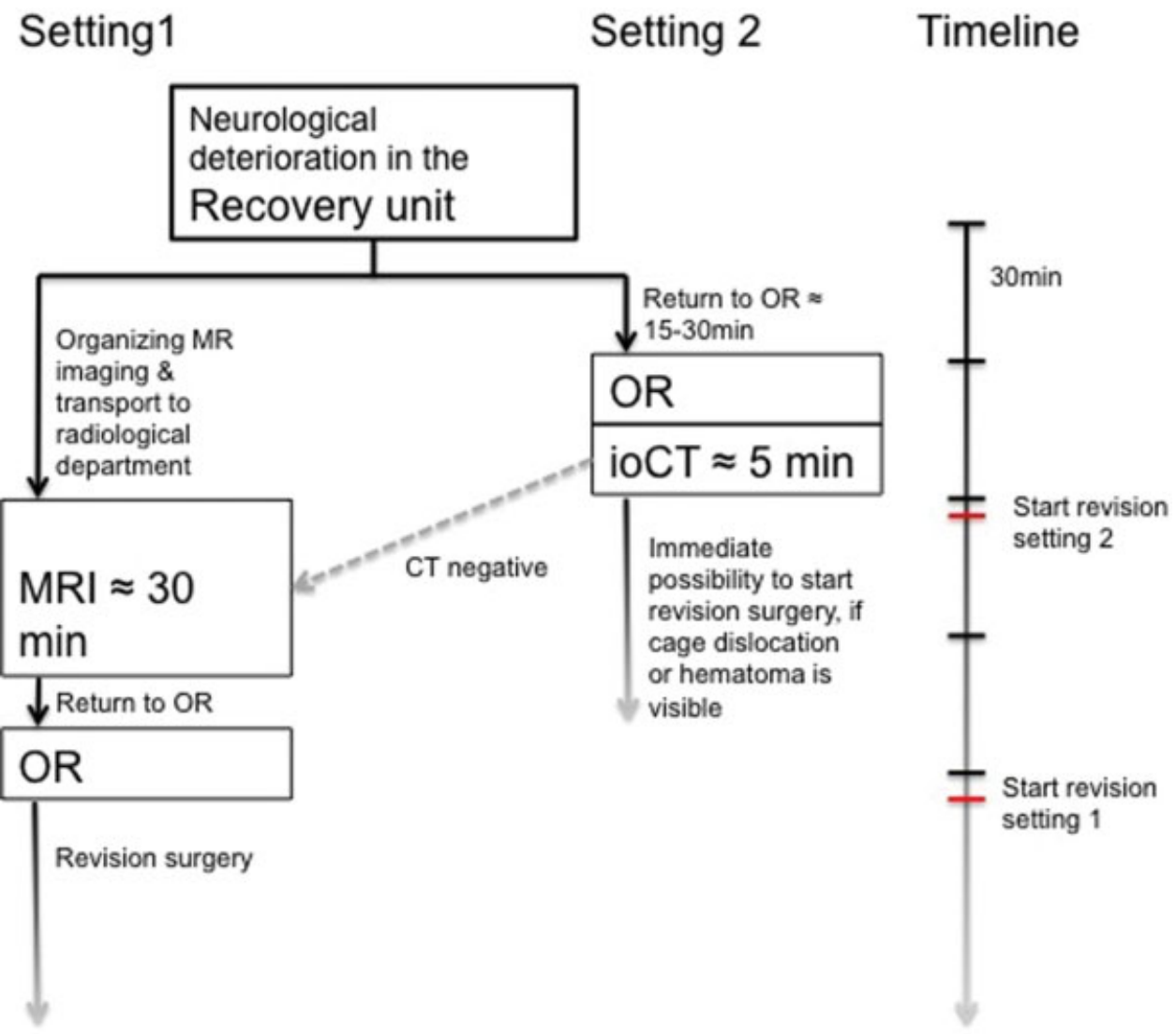

Fig. 4 Flowchart comparing the two possible scenarios if a neurological deterioration develops in the recovery unit. Immediate return to the operating room and rapid imaging using the CT provides sufficient information in case of hematoma or cage dislocation. If the CT gives no explanation for the neurological deficits, an MRI is required additionally, delayed not more than 10 minutes. CT, computed tomography; MRI, magnetic resonance imaging.

After the sudden progressive neurological deterioration, an ischemic process was considered in the differential diagnosis, but in order to not lose time, the CT was performed as first-line imaging in our operating room. If there would not have been an hemorrhagic explanation for the presented deterioration, the loss of time would have amounted to only 10 minutes and an immediate MRI could have been performed for further evaluation thereafter.

Visualization of the processes dorsal to the cervical vertebrae is difficult in a regular ACDF approach because it provides only a narrow space to explore the spinal canal. Even if the approach is amplified in our case with a corpectomy of $\mathrm{C} 5$, it can be difficult to assess the situation at levels superior or inferior to the bony decompression. Even if a sufficient decompression of the SEH seems to be accomplished, an adequate intraoperative imaging modality can reassure the success of the operation. Due to these considerations, several intraoperative imaging tools have been introduced in neurosurgery, each with its advantages and limitations. Intraoperative MRI suites surely provide the best visualization of the spinal cord structures, but it resembles a quite expensive and time-consuming modality. ${ }^{13}$ Furthermore, the method has its limitations after ACDF-procedures because of artifacts of implanted cages, especially in operations with additional anterior plating. Also, intraoperative ultrasound is deviously limited in visualizing structures dorsal the vertebral bodies; it may provide information about the spinal cord after amplified ventral exposure via corpectomy, but especially in degenerative spinal cord compression with ossified posterior longitudinal ligament, it has limited utility due to artifacts from residual ossification and limitation by the bony exposure. ${ }^{14}$ The value of intraoperative threedimensional fluoroscopy has instead proven to give adequate information of (bony) decompression in ACDFprocedures $^{15}$ and is widely used. In comparison to ioCT, however, its image quality is inferior and could miss a hematoma.

The ioCT is widely used in spinal surgery, mostly in fusion-procedures for navigated placement of pedicle screws, as described in numerous studies. But it also provides the possibility of immediate confirmation of adequate decompression in handling $\mathrm{SEH}$ after decompressive cervical procedures. To our knowledge, at this time there are no similar reports in literature about the advantage of ioCT in complication management after cervical spine surgery.

In the presented case, not only the diagnosis of SEH but also repeated $\mathrm{CT}$ during decompression had dramatic clinical relevance. It led to an additional corpectomy one level above (see - Fig. 2), although the senior neurosurgeon (C.T.) had judged the space-occupying hematoma to be removed. 
Adequate treatment of a severe complication could be achieved only by intraoperative imaging in this patient. It must be assumed that stopping surgery after one-level corpectomy would have necessitated a second revision surgery.

\section{Conclusion}

Symptomatic SEH can be readily detected on the operating table, prompting immediate revision surgery in case of a new postoperative neurological deficit. Without this imaging modality, a time delay can be associated with a high risk of persistent deficits. What is more, ioCT allows intraoperative assessment of the adequacy of hematoma removal and decompression. This avoids further neurological damage and repeated revision surgery in case of hematoma spread along the spinal cord.

\section{Disclosure}

The authors report no conflict of interest concerning the materials or methods used in this study or the findings specified in this article.

\section{Approval}

All authors approved this article.

\section{References}

1 Bailey RW, Badgley CE. Stabilization of the cervical spine by anterior fusion. J Bone Joint Surg Am 1960;42-A:565-594

2 Smith GW, Robinson RA. The treatment of certain cervicalspine disorders by anterior removal of the intervertebral disc and interbody fusion. J Bone Joint Surg Am 1958;40-A(3): 607-624

3 Cloward RB. The anterior approach for removal of ruptured cervical disks. J Neurosurg 1958;15(6):602-617
4 Bohlman HH, Emery SE, Goodfellow DB, Jones PK. Robinson anterior cervical discectomy and arthrodesis for cervical radiculopathy. Long-term follow-up of one hundred and twenty-two patients. J Bone Joint Surg Am 1993;75(9): 1298-1307

5 Anderson PA, Sasso RC, Riew KD. Comparison of adverse events between the Bryan artificial cervical disc and anterior cervical arthrodesis. Spine 2008;33(12):1305-1312

6 Hilibrand AS, Carlson GD, Palumbo MA, Jones PK, Bohlman HH. Radiculopathy and myelopathy at segments adjacent to the site of a previous anterior cervical arthrodesis. J Bone Joint Surg Am 1999;81(4):519-528

7 Awad JN, Kebaish KM, Donigan J, Cohen DB, Kostuik JP. Analysis of the risk factors for the development of post-operative spinal epidural haematoma. J Bone Joint Surg Br 2005;87(9):1248-1252

8 Dina TS, Boden SD, Davis DO. Lumbar spine after surgery for herniated disk: imaging findings in the early postoperative period. AJR Am J Roentgenol 1995;164(3):665-671

9 Ikuta K, Tono O, Tanaka T, et al. Evaluation of postoperative spinal epidural hematoma after microendoscopic posterior decompression for lumbar spinal stenosis: a clinical and magnetic resonance imaging study. J Neurosurg Spine 2006;5(5):404-409

10 Aono $\mathrm{H}$, Ohwada $\mathrm{T}$, Hosono $\mathrm{N}$, et al. Incidence of postoperative symptomatic epidural hematoma in spinal decompression surgery. J Neurosurg Spine 2011;15(2):202-205

11 Lin Q Zhou X, Wang X, Cao P, Tsai N, Yuan W. A comparison of anterior cervical discectomy and corpectomy in patients with multilevel cervical spondylotic myelopathy. Eur Spine J 2012; 21(3):474-481

12 Hans P, Delleuze PP, Born JD, Bonhomme V. Epidural hematoma after cervical spine surgery. J Neurosurg Anesthesiol 2003;15(3):282-285

13 Martin XP, Vaz G, Fomekong E, Cosnard G, Raftopoulos C. Intraoperative $3.0 \mathrm{~T}$ magnetic resonance imaging using a dualindependent room: long-term evaluation of time-cost, problems, and learning-curve effect. Acta Neurochir Suppl (Wien) 2011;109:139-144

14 Moses V, Daniel RT, Chacko AG. The value of intraoperative ultrasound in oblique corpectomy for cervical spondylotic myelopathy and ossified posterior longitudinal ligament. $\mathrm{Br} \mathrm{J}$ Neurosurg 2010;24(5):518-525

15 Baldauf J, Müller JU, Fleck S, Hinz P, Chiriac A, Schroeder HW. The value of intraoperative three dimensional fluoroscopy in anterior decompressive surgery of the cervical spine. Zentralbl Neurochir 2008;69(1):30-34 